\title{
Data gathering guidelines for the mapping of environmental sensitivity to oil spill of the Italian coastlines
}

\author{
F. Cumo ${ }^{1}$, F. Cinquepalmi ${ }^{2} \&$ D. Astiaso Garcia ${ }^{1}$ \\ 'Department of "Fisica Tecnica", Rome University "Sapienza", Italy \\ ${ }^{2}$ Member of the Italian National Technical Board for Sea Protection and \\ Sustainable Maritime Traffic, Italy
}

\begin{abstract}
This paper is part of a project of the Fisica Tecnica Department of "Sapienza" University of Rome and the Italian Ministry for Environment, Land and Sea, to finalize the mapping of the environmental sensitivity to oil spill of the Italian coastlines. Considering that the final aim of this project is to produce a core set of Environmental Sensitivity Indices (ESI) maps for the Italian system of Marine and Coastal Protected Areas, the plan foresees the involvement of the Italian Coastguard for the "in situ" survey, to gather part of the data required for the compilation of the matrices, which are beforehand designed for the evaluation of Environmental Sensitivity Indices (ESI). Therefore, this paper proposes a set of guidelines for the Coastguard Officers in order to obtain unambiguous data for each protected zone and surrounding areas. In particular, the guidelines define univocally the various coastal typologies included in the context of the Mediterranean coastlines, giving a shoreline classification in terms of the sensitivity to oil spills, taking into consideration a number of natural, physical, biological and human factors. The guidelines include for each coastal typology: a short physical description, a possible prediction of oil diffusion and a possible response. Those guidelines have been successfully tested during an "in situ" survey, in the coastlines of Cilento and Vallo di Diano National Park. Moreover, the prosecution of the project will offer a clear system for pinpointing ecological, landscape and cultural priorities, for the realization of an effective protection to the areas considered most vulnerable to hydrocarbons spills.
\end{abstract}

Keywords: integrated coastal zone management, hydrocarbons pollution and oil spills, Environmental Sensitivity Index (ESI) maps, risk assessment. 


\section{Introduction}

During the year 2006, the Department of Fisica Tecnica of "Sapienza" University of Rome and the Direction General for Natures Protection of the Italian Ministry for the Environment, Land and Sea have drawn up a method for the evaluation of environmental sensitivity indices (ESI) to marine hydrocarbons pollution based on the elaboration of three appropriate matrices (for natural coastlines, for water stretches and for artificial features) [1], following the general methodology elaborated in a previous similar work for the Lagoon of Venice, coordinated by Prof. A. Zitelli of the University of Architecture IUAV in 1998 [2].

This methodology proposes to characterize every natural or artificial, coastal or marine spot with en ESI number that expresses the respective sensibility value to an eventual contact with hydrocarbons, in terms of contact probability and entity and irreversibility of the damage in question.

This method has been successfully tested in 2006 during the elaboration of the ESI maps of the Submerged Archaeological and Natural Protected Area of Baia in the gulf of Pozzuoli (Naples) [3].

Since the main objective of the project is to apply ESI maps methodology to the overall Italian system of Marine and Coastal Protected Areas, the plan foresees the involvement of the Italian Coastguard, responsible for the marine survey in Italy, for the "in situ" survey, to gather part of the data required for the compilation of the matrices.

However, in order to obtain unambiguous data for each coastal Protected Area, it has been necessary to elaborate a guidelines package in order to provide the Coastguard Officers with useful information for the development of correct field work.

The package firstly contains a guidelines manual that includes a short description of the methodology for the evaluation of ESI values and a morphological/typological classification of the Mediterranean shorelines, with special attention to the characteristics that have an influence on oil diffusion to the sea, the possible response actions and the entity of the damage. Secondly, the package includes different formats to fill in all the data gathered, and a short additional documentary, filmed during the 2007 field work, with the aim to help officers in the realization of accurate field work.

All the metadata gathered by the Coastguard Officers will be sent to the technical and scientific staff of the Fisica Tecnica Department for elaboration and implementation with other scientific information obtained by bibliographic investigations and eventually "ad hoc" field surveys.

Afterward, the overall data will be utilized by the scientific staff for the elaboration of the ESI values, and the implementation of a GIS (Geographical Information System) database that will includes the ESI maps and other ecological, geomorphological and anthropic activities information for the coastlines.

In order to validate the efficiency of the guidelines, a field test has been conducted in the marine and coastal areas of the Cilento and Vallo di Diano 
National Park [4] by the staff of the Fisica Tecnica Department with the Italian Ministry of the Environment, Land and Sea, and in collaboration with the local Italian Coastguard units.

\section{Natural shorelines classification}

The US Government has already drawn up sensitivity maps covering all the Country's sea and Great Lake coastlines, thanks to work carried out by the NOAA (National Oceanographic and Atmospheric Administration) [5]. Since the 1970s NOAA has developed a method for creating environmental sensitivity indices based on the classification of the various coastlines in whole numbers from 1 to 10, giving a higher number to more vulnerable coastal types, identified by different colours [6].

Within the guidelines, we have reported a classification of the coastal types similar to the one carried out by the NOAA, but with specific reference to the peculiar cultural and historical characteristics of the Mediterranean shorelines. Moreover, as the ESI values exposed here derive from the matrix calculation of individually variables components, a single score for each coastal type has not been matched.

To facilitate the data collection, the natural coastlines were then divided into three macro categories identified in matrix one: cliffs, rocky shores and beaches or mudflats.

For each coastal category the following have been carried out: a physical morphological description, a presentation of the possible biological scenarios present in that category, and a short exposition about the predicted oil behaviour with response considerations.

\subsection{Cliffs}

Cliffs are characterized by a vertical intertidal zone, with a slope usually greater than 30 degrees, and a regular exposure to high wave energy. Sediment accumulations are uncommon and ephemeral because waves quickly remove debris slumped from eroding cliffs.

In the Mediterranean areas, cliffs can host biocenosis composed of eventually protected sessile organisms (Lithophaga lithophaga), and often represent an essential habitat for some bird species such as gulls (Larus spp.), falcons (Falco spp.) and terns (sterna spp.).

In fact, the presence of oil in the cliff areas can lead to a huge cause of mortality in sea birds and drastically reduce the hatching of eggs [7].

Regarding the oil action, the hydrocarbons are often held offshore by waves reflecting off the step cliffs. In addition, the impermeable substrate doesn't allow subsurface penetration, and consequently any oil that is eventually deposited could be rapidly removed from exposed faces by wave action.

However, more resistant oils could remain as a patchy band at or above the tide line, with consequent impacts to intertidal communities, especially when a heavy concentration of hydrocarbons comes ashore very swiftly. 
Usually clean up operations are not required, but when necessary, the access can be difficult and dangerous.

\subsection{Rocky shores}

Rocky coastal areas marked by an angle of slope lower than that of cliffs belong to this category. Rocky shores may support large populations of animals and plants, such as organisms living in rock pools (for example, macroalgal communities or barnacles and other arthropods species). These biocenosis, sometimes composed of rare or endemic species, evolved by specializing to live in extreme conditions caused by sudden changes of environmental variables, such as temperature and salinity, but they are not able to tolerate a potential presence of hydrocarbons in their habitat [8].

The oil action depends on the typology of the substrate, which can be impermeable with no potential for subsurface penetration over much of the intertidal zone, or composed of boulders, granules, pebbles and cobbles.

In both cases, heavy accumulations of pooled oil and all oiled debris should be removed quickly, because the impacts to attached organisms can be severe.

In the impermeable rocky surface, the persistence of oil sediments is usually short-term, except in the larger sediment accumulations. However, biological impacts can be immediate and severe, particularly if oil slicks cover pool communities.

In a second possible option, oil could penetrate into the crevices causing long term contamination of the subsurface where it cannot be reworked by any natural process except extreme storms. During the necessary clean up operations, because of slow sediment replenishment rates, sediment removal should be limited as much as possible.

In the case of sheltered rocky shores, the lack of high wave energy slows down the natural removal rates of hydrocarbons, therefore it increases the necessity of clean up operations.

\subsection{Beaches and mudflats}

These shorelines are characterized by a low slope angle and a thin size of substrate subject to regular reworking by waves.

Beach fauna can vary in type and density, with many mobile surface, burrowing and interstitial species, while the vegetation becomes considerable mainly with the presence of dunes.

Heavy oil accumulations usually cover the entire beach intertidal surface, and the maximum penetration of hydrocarbons is about $10 \mathrm{~cm}$ into fine grained sand and $15 \mathrm{~cm}$ into medium grained sand.

Organisms living in the beach may be killed by smothering or lethal oil concentrations in interstitial water.

Due to the loosely packed and permeable nature of the coarse grained sand beaches, clean up is more difficult for these kinds of beaches than for finer grained ones, because equipment tends to grind oil into the substrate; therefore, 
special care must be exercised at all times while using heavy equipment in order to prevent mixing oil deeper into the beach sediments.

Manual clean up, rather than road graders and front-end loaders, is advised where feasible to minimize the volume of sand removed from the shore. In fact, removal of sediment should be limited as much as possible to avoid erosion problems on the beach in the future.

It is also important to prevent the mixture of oil deeper into the sediments by operational movements in the surface.

\section{Data gathered}

The guidelines include a chapter with an explanation of the correct way to realize the data collection as appropriate to the methodology and a set of three different kinds of formats for the registration of the information in the field. The first format has been drawn up for the acquisition of data concerning natural coastlines, the second one is required for the registration of artificial features along shorelines, while the last format has been drawn up for water stretches data. Every format contains a space for registering the coordinates of each significant point, identified through d-GPS (differential GPS) and subsequently elaborated with a specific software (figure 1).

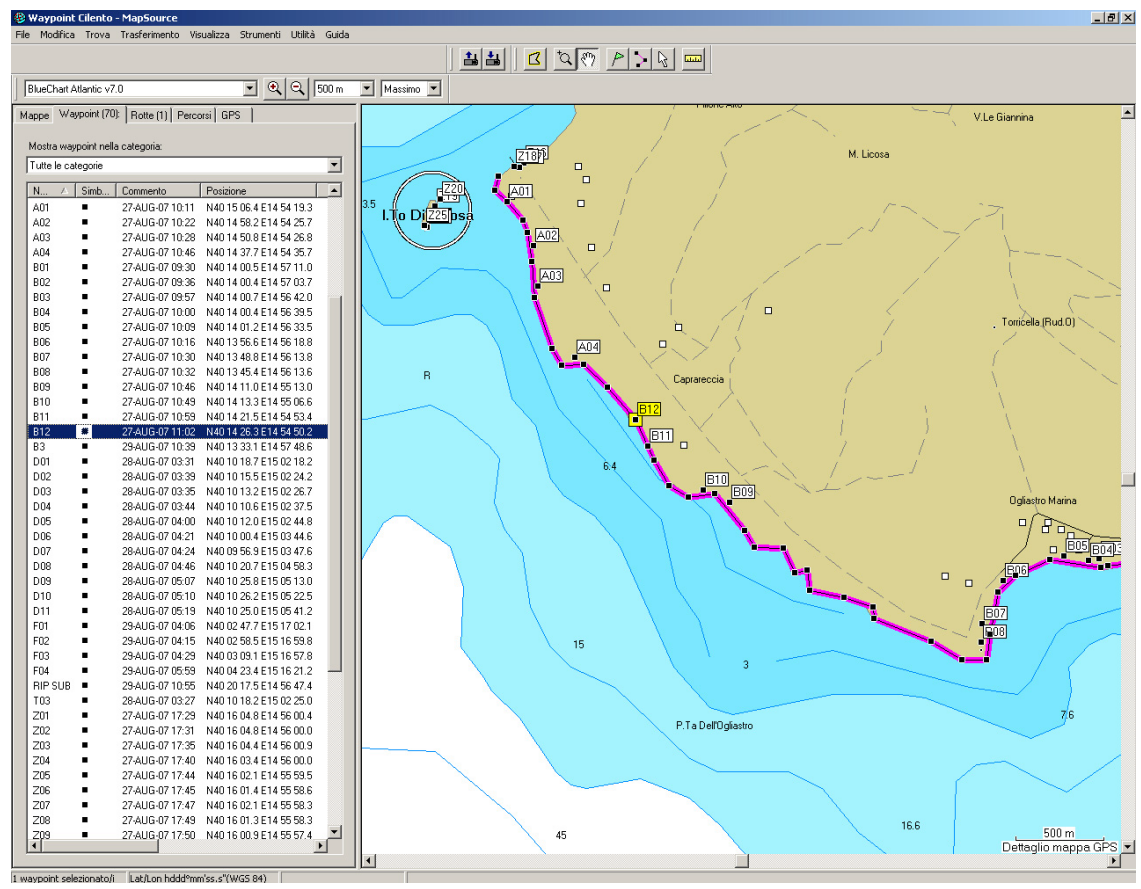

Figure 1: Example of the use of Map Source-bluechart software for the cartographic interface of the points recorded by GPS equipment. 
The effectiveness of the guidelines has been successfully tested in the pilot project for the elaboration of ESI maps in the Cilento and Vallo di Diano National Park coastlines.

The field work has been carried out jointly by the researchers of the "Fisica tecnica" department and the local units of Italian Coastguards, under the supervision of the Directorate General of Nature protection of the Italian Ministry of the Environment, Land and Sea, with the aim of following the correct application of data gathering processes.

The metadata obtained during this field work have been elaborated by the "Fisica Tecnica" Department technicians and implemented with other scientific/bibliographic information referred to the area [9], for the creation of a GIS database that includes the ESI maps of the park coastlines and coastal waters (figure 2).

\section{Conclusions}

The pilot project previously described confirmed that the elaborated guidelines are a valid instrument for obtaining correct data gathered from shorelines information through the help of the local Coastguards.

Consequently, it will be possible to apply the same protocol for the elaboration of ESI maps of the Italian Marine Protected Areas System.

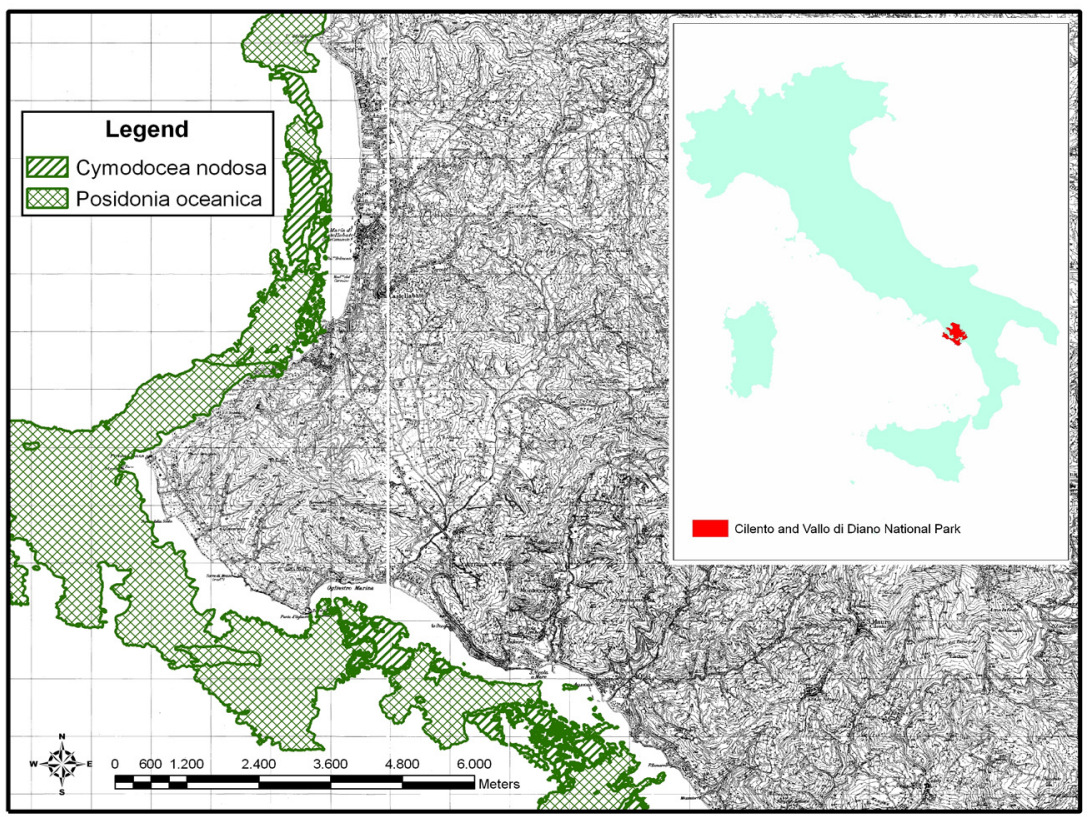

Figure 2: GIS cartography of the marine phanerogams grasslands in the Cilento and Vallo di Diano National Park sea coastal waters. 
The follow up of the project will involve the application of the methodology in the overall Italian system of Marine and Coastal Protected Areas, with the substantial result in quite a short time of the achievement of the overall Italian ESI maps for the identification of those areas that are particularly vulnerable to an accidental oil spill, for environmental, landscape or cultural priorities.

Therefore, it will be possible to plan preventive protection actions based on a global vision of the entire national shorelines.

The establishment of a reliable and accurate coastal mapping system is not only essential for the preparation of an effective national contingency plan against oil spills, but it could also represent a valid instrument to help in the application of the integrated coastal zone management principles.

\section{References}

[1] Gugliermetti F., Cinquepalmi F., Astiaso Garcia D. The use of environmental sensitivity indices (ESI) maps for the evaluation of oil spill risk in Mediterranean coastlines and coastal waters. Third International Conference on Sustainable Development and Planning. WIT Transaction on Ecology and the Environmental. WIT Press, 2007.

[2] Cinquepalmi F., Schiuma D., Tagliapietra D., Benedetti C. and Zitelli A., Prevention of hydrocarbons sea pollution: Sensitivity Index Maps for the Venice Lagoon as integral component of oil-spill contingency planning and response. Environmental Coastal Regions. WIT Transaction of Ecology and the Environmental volume 25. WIT Press, 1998.

[3] Gugliermetti F., Cumo F., Monaco A. The elaboration of environmental sensitivity indices (ESI) maps of the Marine Protected Area of Baia. Second International Conference on Safe 2007 - Safety and Security Engineering. Wessex Institute of Technology Press, 2007.

[4] Ministero dell'Ambiente e della Tutela del Territorio e del Mare. Parchi d'Italia. Il Sistema delle aree protette. Carsa Edizioni.

[5] National Oceanic \& Atmospheric Administration (NOAA) web site: http://response.restoration.noaa.gov/.

[6] National Oceanic \& Atmospheric Administration (NOAA), National Ocean Service, Office of Response and Restoration. Sensitivity of Coastal Environments and Wildlife to Spilled Oil. Hawaii Atlas vol. I - II. 2001.

[7] Cognetti G. \& Cognetti G. Inquinamenti e protezione del mare. Calderini Editore, 1992.

[8] Della Croce N., Cattaneo Vietti R., Danovaro R. Ecologia e protezione dell'ambiente marino costiero. Utet libreria, 1997.

[9] Russo F., Di Stefano F. Realizzazione di una procedura di analisi multicriterale per la gastione integrata della fascia costiera cilentana. APAT (Agenzia per la Protezione dell'Ambiente e per i Servizi Tecnici), Museo Vivo del Mare, Università degli Studi di Napoli "Parthenope". 